\title{
Dose Optimization to Minimize Radiation Risk for Children Undergoing CT and Nuclear Medicine Imaging Is Misguided and Detrimental
}

\author{
Jeffry A. Siegel ${ }^{1}$, Bill Sacks ${ }^{2}$, Charles W. Pennington ${ }^{3}$, and James S. Welsh ${ }^{4}$ \\ ${ }^{I}$ Nuclear Physics Enterprises, Marlton, New Jersey; ${ }^{2}$ U.S. Food and Drug Administration (retired), Green Valley, Arizona; ${ }^{3}$ NAC \\ International (retired), Norcross, Georgia; and ${ }^{4}$ Department of Radiation Oncology, Stritch School of Medicine, Loyola University, \\ Maywood, Illinois
}

\begin{abstract}
A debate exists within the medical community on whether the linear no-threshold model of ionizing radiation exposure accurately predicts the subsequent incidence of radiogenic cancer. In this article, we evaluate evidence refuting the linear no-threshold model and corollary efforts to reduce radiation exposure from CT and nuclear medicine imaging in accord with the as-low-as-reasonablyachievable principle, particularly for children. Further, we review studies demonstrating that children are not, in fact, more radiosensitive than adults in the radiologic imaging dose range, rendering dose reduction for children unjustifiable and counterproductive. Efforts to minimize nonexistent risks are futile and a major source of persistent radiophobia. Radiophobia is detrimental to patients and parents, induces stress, and leads to suboptimal image quality and avoidance of imaging, thus increasing misdiagnoses and consequent harm while offering no compensating benefits.
\end{abstract}

Key Words: radiological imaging; linear no-threshold; ALARA; dose optimization; radiophobia; children

J Nucl Med 2017; 58:865-868

DOI: 10.2967/jnumed.117.195263

$\mathbf{T}$ here is a great divide over exposure to low-dose radiation. Ionizing radiation is thought by many to cause cancer, based on uncritical acceptance of the linear no-threshold model (LNT) despite absence of valid evidence for doses below 100-200 mGy (1). Moreover, the LNT extrapolation from high- to low-dose radiation has proven empirically false and decidedly harmful to millions of people with respect both to medicine and to government policies regarding nuclear power plants (1-4). Herein, we argue that lowdose and low-dose-rate radiation does not cause-but in most people helps prevent-cancer, as shown by sound, empiric, experimental and observational studies. We also briefly summarize the research showing the falsehood of LNT and its detrimental consequences.

This article was prompted by an all-day categoric seminar that will take place this month at the SNMMI annual meeting. The program

Received Apr. 24, 2017; accepted Apr. 24, 2017.

For correspondence contact: Jeffry A. Siegel, Nuclear Physics Enterprises, 4 Wedgewood Dr., Marlton, NJ 08053.

E-mail: nukephysics@comcast.net

Published online May 11, 2017.

COPYRIGHT (C 2017 by the Society of Nuclear Medicine and Molecular Imaging. for "Radiation Dose Optimization in Pediatric Nuclear Medicine" advertises 13 presentations on radiation risk in children, absorbed dose estimation, dose reduction, and radiopharmaceutical guidelines. This seminar will focus on communicating a risk that is, in fact, fictitious; on emphasizing a dose reduction that is, consequently, needless; on achieving a resulting dose estimation enhancement that is superfluous; and on improving activity-administration guidelines. The underlying intent of lowering future cancer risk, although desirable, goes astray, as the premise is based on the erroneous LNT and the resulting as-low-as-reasonably-achievable principle (ALARA). The seminar further aims at procedure standardization in pediatric nuclear medicine, with which we have no quarrel. Although more accurate dosimetry can improve guidelines for radiopharmaceutical activity administration and is a reasonable pathway to optimization of image quality, it is wholly misguided for radiation protection purposes.

LNT and its corollary, ALARA — which are based on a link between absorbed radiation (no matter how low the dose or dose rate) and future cancer probability - are defended despite their proponents' disarming admission that because of the low-doseradiation signal-to-noise problem, there is no evidence, nor can there be any, favoring LNT for low doses. Proponents often defend their reliance on LNT on the grounds that even if there is a dose threshold below which there is no harm, LNT's consequent overestimation of harm errs on the side of caution. Seemingly sound, this application of the precautionary principle ignores empiric low-dose-radiation research while offering no refutation, as well as denying responsibility for the radiophobia, with its attendant harms, generated by LNT and the concomitant result that patients are deprived of the probable cancer-inhibiting effects of low-dose radiation $(1,4)$.

The precautionary principle is useful only if action to control the feared agent has no, or less harmful, side effects. However, for radiologic imaging, the negative consequences of radiophobiabased dose reduction exceed the putative harms of radiation. These consequences include misdiagnosis due to both imaging avoidance and nondiagnostic image quality, as well as use of alternative imaging procedures, such as longer-duration MRI studies, that have a higher sedation risk for children, as we have reported (1). The harmful consequence of misdiagnosis- the need to resort to exploratory surgical alternatives-is greater than the LNT-based fictitious prediction of harm and more immediate than any future cancer risk that is putatively avoided by ALARA dosereduction strategies (5). 
A worldwide movement has arisen in response to the scientifically unsupportable call to decrease dose. We concentrate here on the pediatric CT and nuclear medicine dose "optimization" efforts generated by the Image Gently Alliance for children, a more socially and medically vulnerable population. The efforts aim not only at improving diagnostic quality, a laudable objective, but also at lowering the fictitious risk of future cancer. Recognizing the antagonism between these contradictory objectives, Dr. Donald Frush, chair of the Image Gently Alliance, states that the Alliance seeks to provide balance between them, yet he disclaims any responsibility of the Alliance for the media-driven radiophobia (6). Reasonable as this aim might be if, in fact, there were a risk of cancer from CT or nuclear medicine imaging, balance between diagnostic benefit and cancer risk can no longer claim innocence in the absence of such risk. Balance between truth and falsehood is no balance at all.

\section{CHILDREN ARE NOT MORE RADIOSENSITIVE THAN ADULTS}

The focus on children is founded on their social vulnerability and on a false biologic assertion that children are more radiosensitive than adults. This assertion is based both on an indisputable fact and on a false assumption: the fact that more rapid cell division leads to greater mutation opportunities, and the assumption that increased mutations entail increased cancer risk. But, like LNT itself, the assumption ignores evidence-supported adaptive responses that either repair mutations through enhanced repair enzymes or remove the unrepaired cells by apoptosis or, most importantly, the immune system $(1,3,7)$. In the absence of these evolution-provided naturally selected defenses, the far more frequent (by about a million-to-one) endogenous mutations in almost every cell-due to reactive oxygen species from normal metabolism-would produce cancer minute by minute. Mutations or epigenetic changes are necessary, but not sufficient, to produce clinical cancer.

Most importantly, Preston et al. studied Japanese atomic bomb survivors who were younger than $6 \mathrm{y}$ at the time of the bombing (8). The results indicated no significant difference in adult-onset solidcancer incidence between the control group and the group that received acute exposure of up to $200 \mathrm{mSv}$ as children. CT and nuclear medicine imaging apply doses that are at least an order of magnitude below that figure. Thus, there is epidemiologic evidence that children are not more radiosensitive to such imaging.

In addition, relevant evidence from a study on individuals undergoing CT examination indicates that at low radiation exposures, initial radiation-induced damage is generally repaired or eliminated in a matter of hours by the body's adaptive responses. Löbrich et al. (7), for example, showed that, within minutes, exposure to radiation from CT scans induces an increase in DNA doublestrand breaks compared with pre-CT levels. But these double-strand breaks are subsequently repaired. In fact, in all but one patient, the double-strand breaks were repaired to less than the initial (pre-CT) background level at some time between 5 and $24 \mathrm{~h}$, suggesting that the low-dose CT exposure induced repair of the preexisting and ongoing endogenous DNA damage as well as repair of the radiogenic damage. This is evidence of a beneficial (hormetic) effect of low-dose ionizing radiation and argues against radiogenic causation of either solid cancer or leukemia in children or adults.

Furthermore, in older children the immune system, once established, is more vigorous than that in adults; its efficiency declines with age, and low-dose radiation has been shown to stimulate the immune system to reduce cancer rates (9). Fahey et al., nevertheless, claim it is prudent to strive for optimal administered activity in children by using a patient-size-related dose, defining optimal as the lowest dose that still provides the diagnostic information necessary for proper care (10). Although an insufficient dose has resulted in nondiagnostic studies, this trade-off ignoring the body's proven defensive responses is thought to be necessary.

As with the study of Preston et al. (8), much of the current thinking about the risks of ionizing radiation is based on certain interpretations of the Hiroshima/Nagasaki life-span study. As we have reported (1), these data, properly interpreted, invalidate LNT. Supportively, the French Academy of Sciences (11) reported that these data provide evidence for protective, adaptive responses and no valid evidence for harm below 100 mGy. Despite this contrary evidence, report VII from the National Academy of Sciences' Committee on the Biological Effects of Ionizing Radiation (BEIR committee) (12) still endorsed LNT for solid cancers and, further, attributed a risk 2-3 times higher in children. Unaware perhaps of the BEIR committee's distortion of its own cited data, most radiologists and nuclear physicians look to this widely regarded voice of authority. Indeed, we have found and revealed BEIR VII's egregious misquoting of one of its chosen sources that actually came to the opposite conclusion, in line with our contention (4).

\section{VOICES OF "AUTHORITY" DO MORE HARM THAN GOOD}

Unfortunately, many imaging professionals do not read scientific literature critically. For their understanding of LNT, ALARA, and dose optimization, they rely on "expert" opinion, as offered in reports by the BEIR committee, the International Commission on Radiological Protection, the National Council on Radiation Protection and Measurements, and others that mutually reinforce each other. Further, many investigators discount the work of those with whom they disagree rather than even attempting to refute it, instead appealing to citations from these authoritative organizations as though they were established fact rather than the mere opinion that closer examination proves them to be.

As we have reported $(1,4)$, there are two large epidemiologic studies that have been frequently cited as evidence to support the claim that children are at an increased risk of cancer due to CT. But according to the United Nations Scientific Committee on the Effects of Atomic Radiation, and even Dr. John Boice (13), president of the National Council on Radiation Protection and Measurements, the results of these studies are likely untrue. Inaccurate dosimetry and implausible risk estimates are apparent; association is conflated with causation; and medical indication rather than radiation is likely the source of the apparent association (i.e., reverse causation). Further, again as we reported and as underscored by Dr. Boice, two more recent epidemiologic studies, in France and Germany in 2015, found no significant excess cancer risk in children from CT scans, “... once adjustment was made for conditions that prompted the scan, family history, or other predisposing factors known to be associated with increased cancer risk" (13). Thus, confounding by indication (reverse causation) must be ruled out completely, or observed excess cancer risk may be falsely, and facilely, attributed to CT exposure.

Dr. David Brenner et al. (14) published an article in 2001 that was widely promoted by the media, accelerating the prevalence and intensity of radiophobia. This article has been cited in the scientific literature over a thousand times since. On the basis of 
an LNT calculation involving unsupported parameters with significant uncertainties, the authors projected that approximately 500 children under the age of $15 \mathrm{y}$ would eventually die of CT-caused cancer. Some years later, in the New England Journal of Medicine, the same Brenner, with Hall, predicted that up to $2 \%$ of all cancers in the United States "could" be due to CT scans (15). Importantly, these erroneous and irresponsibly fear-amplifying predictions were based on CT doses that were completely unknown for individual patients and were based on risk estimates from the Hiroshima/ Nagasaki life-span study cohort. These risk estimates are not applicable to children undergoing CT examinations in the United States, for one thing because it is most unlikely that the radiation will have played a causal role in children who do develop cancer after a CT scan. Further, whatever the cancer rates in bomb survivors, they were also subjected to the confounding factors of severe trauma and deprivation, which are known to interfere with immune system function and hence enhance susceptibility to all sorts of ailments.

Brenner's group subsequently reported wholly unjustified conclusions again promoting widespread radiophobia, this time with respect to double-strand breaks in only 3 pediatric patients after undergoing CT examinations (16). Even acknowledging the finding of Löbrich et al. (7) that repair or removal of initial damage was expected within several hours of exposure, this group obtained data only at $1 \mathrm{~h}$ after the CT scans, which, as expected, demonstrated increased double-strand breaks compared with preCT levels. Yet the group concluded that its findings supported LNT at very low doses in young children and that "even very low ionizing radiation exposure relevant to diagnostic CT exposure can leave a mark in the somatic DNA," significantly and irresponsibly omitting the qualifier "for perhaps a few hours." Astonishingly, the group recommended that, based on this admittedly incomplete evidence, "When possible, CT exams should be limited or avoided by possibly applying non-ionizing radiation exposure techniques such as US or MRI." Such alternatives are sometimes medically warranted, but their use should be based only on their being the most appropriate imaging modality, not on their producing a lower radiation exposure.

\section{THE SEARCH FOR ACCURACY IN THE ABSENCE OF VALIDITY}

The dose-optimization movement in nuclear medicine has prompted intense de novo efforts to develop numerous complex anatomic phantoms and better pharmacokinetic data and models to more accurately estimate absorbed doses for diagnostic procedures (as opposed to therapeutic procedures) (17). Furthermore, the current consensus guidelines for administered radiopharmaceutical activity are considered inadequate and in need of updating. This more precise and labor-intensive endeavor is deemed necessary to improve the practice of pediatric nuclear medicine imaging by providing excellent image quality at the lowest radiation dose possible, but the obsession over lowering radiation dose is a futile and laborious attempt to minimize what is, in fact, a nonexistent risk.

As one example of the relative superfluity of such precision, much effort has already been expended on assessing pediatric use of ${ }^{99 \mathrm{~m}} \mathrm{Tc}$-dimercaptosuccinic acid, for which the highest effective dose per imaging examination is only about a third of the average annual natural background radiation dose-3.1 $\mathrm{mSv}$ - received per person in the United States, with the average background dose elsewhere in the world ranging to up to 80 times greater. This effective dose, therefore, is well within the hormetic zone; that is, below the threshold for harm.

The original aim of the radiopharmaceutical guidelines was to reduce the variability of administered activities. This aim is praiseworthy for better standardization of image quality but irrelevant for radiation protection purposes. The need for better accuracy in dose estimation to further minimize a nonexistent risk based solely on the erroneous LNT is entirely misdirected and, as a serious unintended consequence, serves only to amplify long-standing public and professional radiophobia.

\section{CONCLUSION}

Many critics argue for termination of the fictitious LNT, along with its epiphenomenal ALARA and the Image Gently Alliance, but must contend with a well-ensconced paradigm—one backed by worldwide advisory organizations and regulatory agencies whose overlapping members have invested reputations and labor. Critics must fight not only for predominance of empiric evidence but also against concepts such as the widely accepted yet misapplied justification and optimization principles of the International Commission on Radiological Protection (1). Eliminating any and all diagnostic medical procedures that are not clinically warranted is important. But attempting to lessen fictitious risk by lowering dose in studies that are clinically warranted is a misapplication of the principles. It is protection from radiophobia, rather than from low-dose radiation, that will benefit both children and adults.

The Image Gently Alliance and myriad international affiliated groups harm patients and parents by promoting radiophobia while offering fictitious protection against nonexistent risks. We do not suggest a cavalier approach by discounting dose optimization, but only in service of diagnostic quality. Public misperceptions should not be disregarded or ignored, but neither should they be endorsed; rather, they must be corrected through accurate information. The public's trust in medical practitioners can be attained only if we convince the public and physicians alike that there is no harm in radiologic imaging.

Accurate information about low-dose radiation is the only way to undo fear, as decades of failed alternative approaches and concessions have shown. Although it may seem reasonable to attempt to assuage public fears by accommodating misperceptions through lowering dose despite the absence of harm, such measures only reinforce the misperceptions. Radiophobia is detrimental to patients and parents, induces stress, and leads to suboptimal image quality or avoidance of imaging, both of which produce misdiagnoses. Misperception can be overcome only by rejection of the LNT fiction and its corollary principle, ALARA, and by termination of the Image Gently Alliance.

\section{DISCLOSURE}

No potential conflict of interest relevant to this article was reported.

\section{REFERENCES}

1. Siegel JA, Pennington CW, Sacks B. Subjecting radiologic imaging to the linear no-threshold hypothesis: a non sequitur of non-trivial proportion. $\mathrm{J} \mathrm{Nucl} \mathrm{Med}$. 2017;58:1-6. 
2. Siegel JA, McCollough CH, Orton CG. Point/counterpoint: advocating for use of the ALARA principle in the context of medical imaging fails to recognize that the risk is hypothetical and so serves to reinforce patients' fears of radiation. Med Phys. 2017;44:3-6.

3. Siegel JA, Welsh JS. Does imaging technology cause cancer? Debunking the linear no-threshold model of radiation carcinogenesis. Technol Cancer Res Treat. 2016;15:249-256.

4. Sacks B, Meyerson G, Siegel JA. Epidemiology without biology: false paradigms, unfounded assumptions, and specious statistics in radiation science. Biol Theory. 2016;11:69-101.

5. Siegel JA, Stabin MG. Medical imaging: the challenges of radiation risk assessment. J Nucl Med. 2014;55(12):16N-17N.

6. Frush DP, Goske MJ. Image Gently: toward optimizing the practice of pediatric CT through resources and dialogue. Pediatr Radiol. 2015;45:471-475.

7. Löbrich M, Rief N, Kühne M, et al. In vivo formation and repair of DNA doublestrand breaks after computed tomography examinations. Proc Natl Acad Sci USA. 2005;102:8984-8989.

8. Preston DL, Cullings H, Suyama A, et al. Solid cancer incidence in atomic bomb survivors exposed in utero or as young children. J Natl Cancer Inst. 2008;100:428436

9. Liu SZ. Cancer control related to stimulation of immunity by low-dose radiation. Dose Response. 2006;5:39-47.
10. Fahey FH, Ziniel SI, Manion D, Treves ST. Effects of Image Gently and the North American guidelines: administered activities in children at 13 North American pediatric hospitals. J Nucl Med. 2015;56:962-967.

11. Aurengo A, Averbeck D, Bonnin A, et al. Dose Effect Relationships and Estimation of the Carcinogenic Effects of Low Doses of Ionizing Radiation. Paris, France: Académie des Sciences-Académie nationale de Médecine; March 2005.

12. National Research Council of the National Academies. Health Risks from Exposure to Low Levels of Ionizing Radiation: BEIR VII Phase 2. Washington, DC: The National Academies Press; 2006.

13. Boice JD Jr. Radiation epidemiology and recent paediatric computed tomography studies. Ann ICRP. 2015;44(1, suppl):236-248.

14. Brenner D, Elliston CD, Hall EJ, Berdon WE. Estimated risks of radiation induced fatal cancer from pediatric CT. AJR. 2001;176:289-296.

15. Brenner DJ, Hall EJ. Computed tomography: an increasing source of radiation exposure. N Engl J Med. 2007;357:2277-2284.

16. Halm BM, Franke AA, Lai JF, et al. $\gamma$-H2AX foci are increased in lymphocytes in vivo in young children $1 \mathrm{~h}$ after very low-dose $\mathrm{x}$-irradiation: a pilot study. Pediatr Radiol. 2014;44:1310-1317.

17. Fahey FH, Goodkind AB, Plyku D, et al. Dose estimation in pediatric nuclear medicine. Semin Nucl Med. 2017;47:118-125. 